Research Paper

\title{
DR5 mAb-conjugated, DTIC-loaded immuno-nanoparticles effectively and specifically kill malignant melanoma cells in vivo
}

\author{
Baoyue Ding ${ }^{1,2,3, *}$, Wei Zhang ${ }^{4, *}$, Xin Wu ${ }^{5, *}$, Jeffrey Wang ${ }^{3}$, Chen $\mathrm{Xie}^{3}$, Xuan Huang ${ }^{1}$, \\ Shuyu Zhan ${ }^{1}$, Yongxia Zheng ${ }^{1}$, Yueyan Huang ${ }^{1}$, Ningyin $X^{1}{ }^{1}$, Xueying Ding ${ }^{5}$, \\ Shen Gao² \\ ${ }^{1}$ Department of Pharmaceutics, Medical College of Jiaxing University, Jiaxing, PR China \\ ${ }^{2}$ Department of Pharmaceutics, Changhai Hospital, Second Military Medical University, Shanghai, PR China \\ ${ }^{3}$ Department of Pharmaceutical Sciences, College of Pharmacy, Western University of Health Sciences, Pomona, USA \\ ${ }^{4}$ Department of Pharmaceutics, Shanghai Pulmonary Hospital, Tongji University, Shanghai, PR China \\ ${ }^{5}$ Department of Pharmaceutics, Shanghai General Hospital, Shanghai Jiaotong University, Shanghai, PR China \\ *These authors have contributed equally to this work \\ Correspondence to: Shen Gao, email: liullk@126.com \\ Xueying Ding, email: dingxueying@126.com \\ Baoyue Ding, email: lena_310@163.com \\ Keywords: malignant melanoma, DR5 monoclonal antibodies, dacarbazine, chemoimmunotherapy, active targeting \\ nanoparticles \\ Received: April 25, $2016 \quad$ Accepted: July 19, $2016 \quad$ Published: August 2, 2016
}

\section{ABSTRACT}

We combined chemo- and immunotherapies by constructing dual therapeutic function immuno-nanoparticles (NPs) consisting of death receptor 5 monoclonal antibody (DR5 mAb)-conjugated nanoparticles loaded with dacarbazine (DTIC) (DTIC-NPs-DR5 mAb). We determined the in vivo targeting specificity of DTIC-NPsDR5 mAb by evaluating distribution in tumor-bearing nude mice using a real-time imaging system. Therapeutic efficacy was assessed in terms of its effect on tumor volume, survival time, histomorphology, microvessel density (MVD), and apoptotic index (AI). Systemic toxicity was evaluated by measuring white blood cells (WBC) counts, alanine aminotransferase (ALT) levels, and creatinine clearance (CR).In vivo and ex vivo imaging indicates that DR5 mAb modification enhanced the accumulation of NPs within the xenograft tumor. DTIC-NPs-DR5 mAb inhibited tumor growth more effectively than DTIC or DR5 mAb alone, indicating that combining DTIC and DR5 mAb through pharmaceutical engineering achieves a better therapeutic effect. Moreover, the toxicity of DTIC-NPs-DR5 mAb was much lower than that of DTIC, implying that DR5 mAb targeting reduces nonspecific uptake of DTIC into normal tissue and thus decreases toxic side effects. These results demonstrate that DTIC-NPs-DR5 mAb is a safe and effective nanoparticle formulation with the potential to improve the efficacy and specificity of melanoma treatment.

\section{INTRODUCTION}

Malignant melanoma (MM) is the most aggressive form of skin cancer causing the majority of skin cancerrelated deaths. Its incidence has increased in recent decades worldwide [1]. While adequate resection of the primary cutaneous melanoma leads to an excellent prognosis [2], the prognosis of metastatic melanoma is poor, with a fiveyear survival rate less than $6 \%$ and median survival time less than one year [3]. The most common treatment for the metastatic melanoma is chemotherapy; dacarbazine (DTIC) is the first-line drug [4]. However, DTIC therapy fails in $80-87 \%$ of patients [5], due to its rapid clearance following intravenous administration and the development of drug resistance. Consequently, the clinical application and therapeutic benefits of DTIC are limited [6-8]. Novel therapeutic strategies are urgently needed to improve patient outcomes for malignant melanoma $[9,10]$. 
Its selective toxicity against malignancies makes TNF-related apoptosis inducing ligand (TRAIL) a promising anti-cancer candidate $[11,12]$. TRAIL binds to death receptors 4 and 5 (DR4 and DR5), triggering an extrinsic apoptosis pathway by activating caspase- 8 and subsequently, caspase-3 [13-15]. Many studies indicate that DR5 is highly expressed on the surface of MM cells and therefore can serve as a promising therapeutic target for MM [16-20]. In comparison to TRAIL, agonistic monoclonal antibody against DR5 (DR5 mAb) has been proven to possess potent in vivo antitumor activity with even better stability, lower clearance, and less toxicity [2123]. In early phase clinical trials, monoclonal antibodies targeting DR5 (DR5 mAb) have also been shown to increase the cytotoxicity of conventional chemotherapeutic drugs [24-26].

Recent studies have focused on combining immune-based drugs, such as therapeutic monoclonal antibodies, with chemotherapeutic agents to achieve better MM treatments [27-29]. Given the difference in DR5 expression level between normal and MM cells, DR5 mAbs should be useful for targeted drug delivery. We developed a dual function immuno-nanoparticle formulation (DTIC-NPs-DR5 $\mathrm{mAb}$ ) in our previous study with DTIC encapsulated on the inside and DR5 mAb covalently linked to the NP surface [30]. DTICNPs-DR5 mAb may possess not only the anti-cancer effects of DTIC and DR5 mAb, but also additional favorable pharmacokinetic properties, such as a long systemic circulation time and actively targeted distribution. In our previous in vitro studies, we demonstrated that DTIC-NPs-DR5 mAb specifically targeted and efficiently entered DR5 overexpressing MM cells. Additionally, these immuno-nanoparticles enhanced tumor cytotoxicity, increased cell apoptosis, and decreased non-specific toxicity.

In the current study, the in vivo targeting and therapeutic effect as well as the non-specific toxicity of DTIC-NPs-DR5 mAb were assessed using a malignant melanoma xenograft mouse model. Phosphate buffered saline (PBS), DTIC, DR5 mAb, blank nanoparticles (Blank-NPs), DTIC-loaded nanoparticles (DTIC-NPs) and DR5 mAb-conjugated blank nanoparticles (BlankNPs- DR5 mAb) were used as controls.

\section{RESULTS}

\section{Characterization of the nanoparticles}

As shown in Table 1, the mean diameter of DTICNPs-DR5 mAb was $170.0 \pm 4.1 \mathrm{~nm}$, with an acceptable polydispersity index (PDI) of less than 0.3 , and a zeta potential of $-34.6 \pm 2.3 \mathrm{mV}$. The morphology of the DTIC-NPs-DR5 mAb was determined as described in our previous study [30], they were found to be spherically shaped and moderately uniform. Drug loading (DL) and encapsulation efficiency (EE) of DTIC were $17.8 \pm 0.8$ $\mu \mathrm{g} / \mathrm{mg}$ and $71.7 \pm 2.5 \%$, respectively. The amount of DR5 $\mathrm{mAb}$ conjugated to the nanoparticles was approximately $12.8 \pm 2.4 \mu \mathrm{g}$ DR5 $\mathrm{mAb} / \mathrm{mg}$ nanoparticles as quantified by a protein assay.

\section{Dynamic and specific distribution of the NPs in vivo}

The mAb-FITC modified nanoparticles encapsulating a fluorescence agent (PE) were intravenously injected into tumor-bearing mice via the tail vein. Visible fluorescent distribution of PE in tumor-bearing mice was observed for $24 \mathrm{~h}$ using the IVIS Lumina II small animal in vivo real-time imaging system (Figure 1A). At $3 \mathrm{~h}$ post administration, a clear PE signal was visible at the tumor site. The signal reached its maximum at $6 \mathrm{~h}$, and then gradually decreased. This suggests that the PE-NPs-DR5 mAb-FITC distribute into tissues in a time-dependent manner.

Tumors and other organs were excised and observed by ex vivo imaging $10 \mathrm{~h}$ after intravenous injection. As shown in Figure 1B, fluorescence signals of the unmodified NPs were observed in the tumor, lung and kidneys. Compared to unmodified NPs, the fluorescence signal of mAb-NPs was stronger in the tumor, weaker in the lung and not observed in the kidneys. This finding indicates that the mAb-NPs more specifically accumulated in tumor tissue in vivo than simple NPs did.

\section{In vivo antitumor efficacy}

Tumor weight and volume were determined for the evaluation of antitumor effect. Figure 2 shows the change of tumor volume with time (day) when treated with different agents. It is obvious that the animals treated with DTIC-NPs-DR5 mAb (Group A) show the slowest tumor growth rates and smallest tumor volumes. As shown in Table 2 and Figure 3, compared to PBS control (Group H), no significant change of tumor volume was observed in animals receiving Blank-NPs (Group G), and the tumor volume in the drug administered groups (Groups A-F) was significantly decreased $(p<0.05)$. Moreover, the inhibition of tumor volume using the combination of DITC and mAb (Groups A and B) was superior to monotherapy with DITC or mAb $(p<0.05)$ (Groups C-F). Notably, DTIC-NPs-DR5 mAb treatment exhibited the most significant suppression of tumor growth. Compared with the control Group H, all drug administered groups show significant prolongation of survival time except the DTIC and Blank-NPs groups $(p<0.05)$ (Table 3 and Figure 4$)$. The longest mean median survival was observed in the DTIC-NPs-DR5 $\mathrm{mAb}$ group. 
Table 1: Characterization and drug content of DTIC-NPs-DR5 mAb $(n=3)$

\begin{tabular}{lccccc}
\hline Size $(\mathbf{n m})$ & PDI & $\begin{array}{c}\text { Zeta potential } \\
(\mathbf{m V})\end{array}$ & $\begin{array}{c}\text { DL of DTIC } \\
(\boldsymbol{\mu g} / \mathbf{m g})\end{array}$ & $\begin{array}{c}\text { EE of DTIC } \\
(\mathbf{\%})\end{array}$ & $\begin{array}{c}\text { DL of DR5 } \\
\mathbf{m A b}(\boldsymbol{\mu g} / \mathbf{m g})\end{array}$ \\
\hline $170.0 \pm 4.1$ & 0.29 & $-34.6 \pm 2.3$ & $17.8 \pm 0.8$ & $71.7 \pm 2.5$ & $12.8 \pm 2.4$ \\
\hline
\end{tabular}
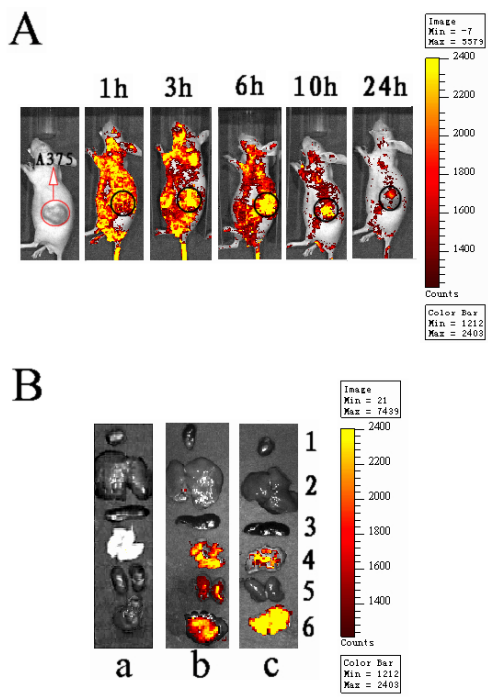

Figure 1: In vivo dynamic and specific distribution of antibody modified NPs. A. Real-time in vivo bioluminescence imaging of PE-NPs-DR5 mAb-FITC in tumor-bearing nude mice at different time points (intravenously inject with $5 \mathrm{mg}$ PE-NPs-DR5 mAb-FITC). B. Representative fluorescence images of dissected organs of nude mice bearing MM sacrificed $10 \mathrm{~h}$ after intravenous injection of different nanoparticles. a: Blank-NPs (control group); b: PE-NPs; c: PE-NPs-DR5 mAb-FITC. 1.heart; 2.liver; 3.spleen; 4.lung; 5.kidneys; 6.tumor. All images were acquired under the same conditions ( $5 \mathrm{mg} / \mathrm{ml}, 0.2 \mathrm{ml} \mathrm{NPs}$ per mouse).

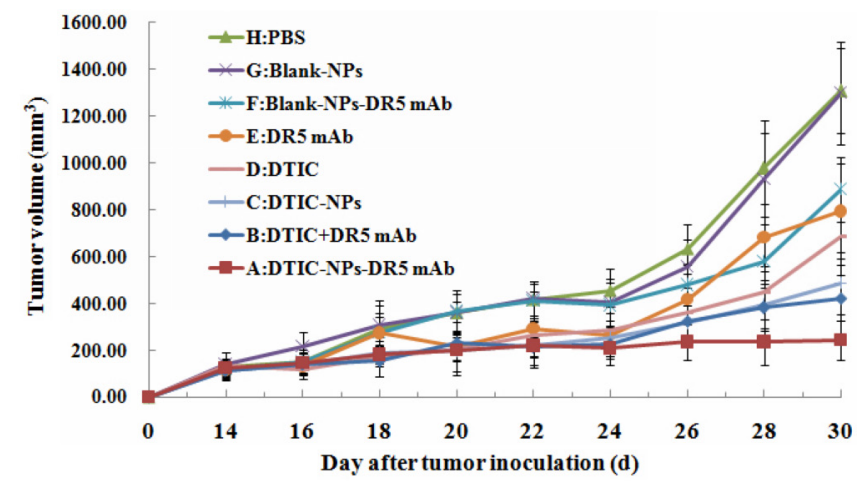

Figure 2: Growth curve of tumor in nude mice in therapeutic experiments. Antitumor agents were given on the 14th day. A: DTIC-NPs-DR5 mAb group. B: DTIC + DR5 mAb group. C: DTIC-NPs group. D: DTIC group. E: DR5 mAb group. F: BlankNPs-DR5 mAb group. G: Blank-NPs group. H: PBS group.

\section{Histological examination, apoptosis index, and vascular count}

For a morphological characterization of the antitumor effect, hematoxylin-eosin staining was used to stain the tumors sections from all groups (Figure 5). High tissue density and vigorous cell growth in tumor tissue were observed in the PBS and Blank-NPs groups. The tissue density decreased in the DTIC treatment (groups $\mathrm{C}$ and $\mathrm{D}$ ) and DR5 mAb treatment (groups E and F). In the combined therapy (groups A and B), cell proliferation decreased and slightly fibrous tissues were observed. In particular, large necrotic regions and extensive necrotic fibrous tissue inside the tumor was detected in Group A with DTIC-NPs-DR5 mAb treatment, indicating the dual therapeutic functional nanoparticles (DTIC-NPs-DR5 $\mathrm{mAb}$ ) possessed tumoricidal activity.

TUNEL staining was carried out to analyze intratumoral apoptosis. In the treatment groups (Groups A - F), apoptotic cells and apoptotic bodies within the cells were 
Table 2: Average tumor weight and tumor growth inhibition rate $(n=6)$

\begin{tabular}{lcc}
\hline Group & Average tumor weight (g) & Tumor growth inhibition rate (\%) \\
\hline A & $0.29 \pm 0.19^{\Delta *}$ & 81.32 \\
B & $0.58 \pm 0.20^{\# *}$ & 63.45 \\
C & $0.82 \pm 0.25^{\# \Delta *}$ & 48.10 \\
D & $0.89 \pm 0.11^{\# \Delta *}$ & 43.53 \\
E & $0.94 \pm 0.13^{\# \Delta *}$ & 40.13 \\
F & $1.01 \pm 0.18^{\# \Delta *}$ & 35.68 \\
G & $1.44 \pm 0.27^{\# \Delta}$ & 8.65 \\
H & $1.57 \pm 0.35^{\# \Delta}$ & - \\
\hline
\end{tabular}

${ }^{\#} p<0.05$, compared with DTIC-NPs-DR5 mAb (group A). ${ }^{\Delta} p<0.05$, compared with DTIC+DR5 mAb (group B). * $p<0.05$, compared with blank control group (group H).

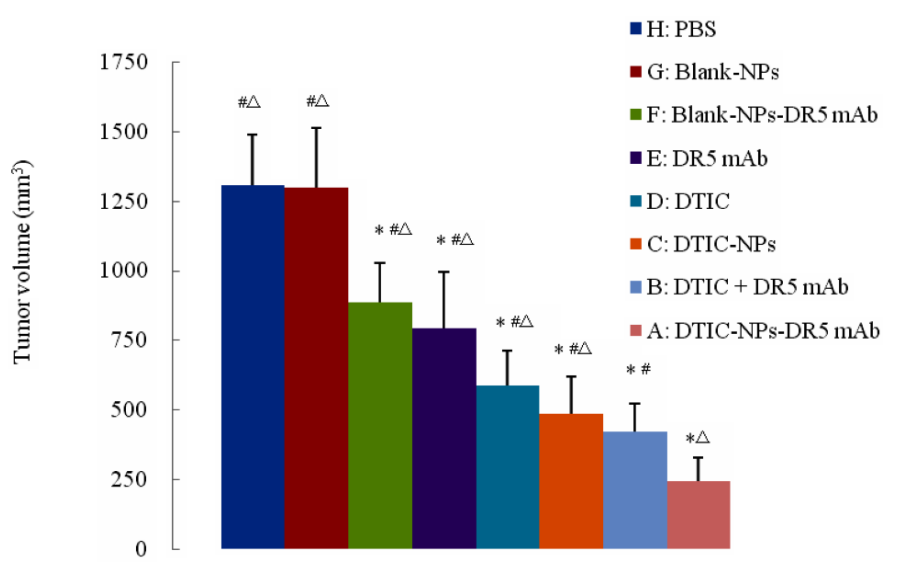

Figure 3: Final tumor volume of tumor-bearing nude mice in therapeutic experiments. ${ }^{\sharp} p<0.05$, compared with DTIC-NPs$\mathrm{DR} 5 \mathrm{mAb}$ (group A). ${ }^{\Delta} p<0.05$, compared with DTIC+DR5 $\mathrm{mAb}$ (group B). ${ }^{*} p<0.05$, compared with blank control group (group H).

observed in the immunohistochemical staining images (Figure 6). DTIC-NPs-DR5 mAb and DTIC+DR5 mAb were more effective in inducing apoptosis than other treatment formulations (B-F) $(p<0.05)$, and DTIC-NPsDR5 mAb was superior to DTIC+DR5 mAb (Figure 7).

Suppression of tumor vasculature was assessed through the detection of MVD as shown in Figures 8 and 9, the suppressive effect of DTIC-NPs-DR5 mAb (Group A) on tumor angiogenesis was more significant than other groups except the DTIC + mAb (Group B) $(p<0.05)$.

\section{Systemic toxicity}

The most common side-effects of DTIC are marrow suppression, and hepatic and renal dysfunction. To evaluate the in vivo toxicity of the formulations, white blood cell number (WBC), alanine aminotransferase (ALT) level and creatinine clearance (CR) in the blood of the tumor-bearing nude mice were determined after treatment. As shown in Table 4 , the WBC in the groups receiving DTIC solution (Groups B and D) was significantly lower than that in the PBS control group (Group H) and normal control group $(p<0.05)$. Meanwhile, DTIC embedded into NPs (Groups A and C) resulted in a normal WBC value, indicating that the pharmaceutical engineering could reduce the marrow suppression by DTIC. Similar results were obtained on ALT. Compared with the control group (Group H) and normal control group, the ALT level was lower in the DTIC solution groups (Groups B and D) $(p<0.05)$, and normal in the DTIC-NPs-DR5 mAb group (Group A). There was no significant change in CR in any groups. These findings indicate that the DTIC-NPs-DR5 $\mathrm{mAb}$ can alleviate the side-effects of DTIC.

\section{DISCUSSION}

In this study, we prepared therapeutic function immuno-nanoparticles (DTIC-NPs-DR5 $\mathrm{mAb}$ ) and evaluated them in vivo. DTIC plays a role for tumoricidal chemotherapeutic activity, and their release from the nanoparticles can be controlled [30]. DR5 mAb on the NP 
Table 3: Means and medians for survival time and the pairwise comparisons analyzed by the Kaplan-Meier method $(\mathrm{n}=10)$

\begin{tabular}{lccccc}
\hline Group & Mean (days) & Median (days) & \multicolumn{3}{c}{ Pairwise comparisons for survival time } \\
\cline { 3 - 5 } & & & $\begin{array}{c}\text { Compared with } \\
\text { group A }\end{array}$ & $\begin{array}{c}\text { Compared with } \\
\text { group B }\end{array}$ & $\begin{array}{c}\text { Compared with } \\
\text { group H }\end{array}$ \\
\hline A & $66.6 \pm 4.2$ & 64.0 & - & $\Delta$ & $* *$ \\
B & $53.5 \pm 3.3$ & 53.0 & $\#$ & - & $* *$ \\
C & 44.0 & $\#$ & $\Delta$ & $* *$ \\
D & $43.9 \pm 2.7$ & 40.0 & $\#$ & $\Delta$ & $p<0.05$ \\
E & $41.8 \pm 1.7$ & 40.0 & $\#$ & $\Delta$ & $*$ \\
F & $41.6 \pm 3.1$ & 41.0 & $\#$ & $\Delta \Delta$ & $*$ \\
G & $40.8 \pm 2.2$ & 36.0 & $\#$ & $\Delta$ & $p<0.05$ \\
H & $37.2 \pm 2.5$ & 36.0 & $\#$ & $\Delta$ & - \\
\hline
\end{tabular}

${ }^{\#} p<0.05,{ }^{\#} p<0.01$, compared with DTIC-NPs-DR5 mAb (group A). ${ }^{\Delta} p<0.05,{ }^{\Delta \Delta} p<0.01$, compared with DTIC+DR5 mAb (group B). ${ }^{*} p<0.05, * * p<0.01$, compared with blank control group (group H).

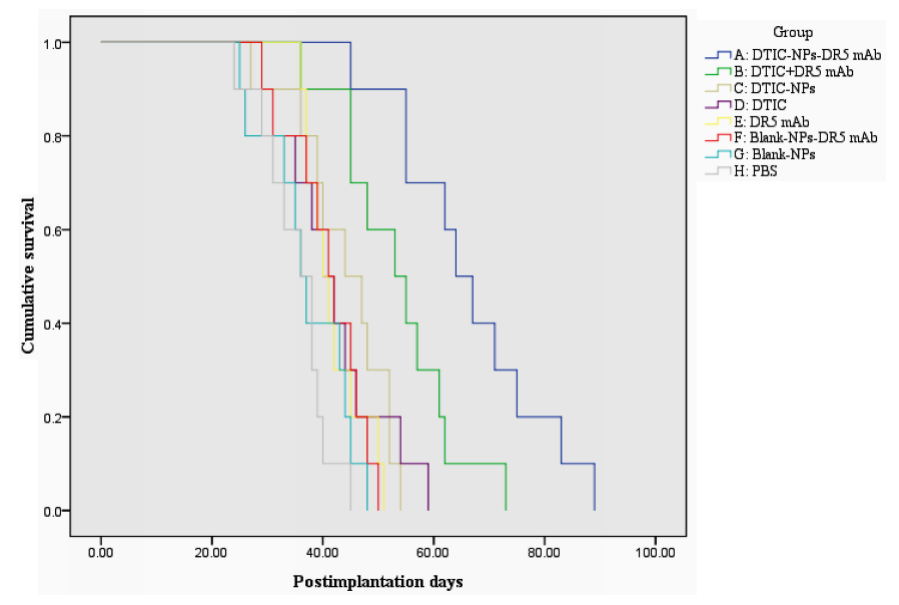

Figure 4: Kaplan-Meier survival curve of tumor-bearing nude mice in therapeutic experiments. A: DTIC-NPs-DR5 mAb group. B: DTIC + DR5 mAb group. C: DTIC-NPs group. D: DTIC group. E: DR5 mAb group. F: Blank-NPs-DR5 mAb group. G: BlankNPs group. H: PBS group.
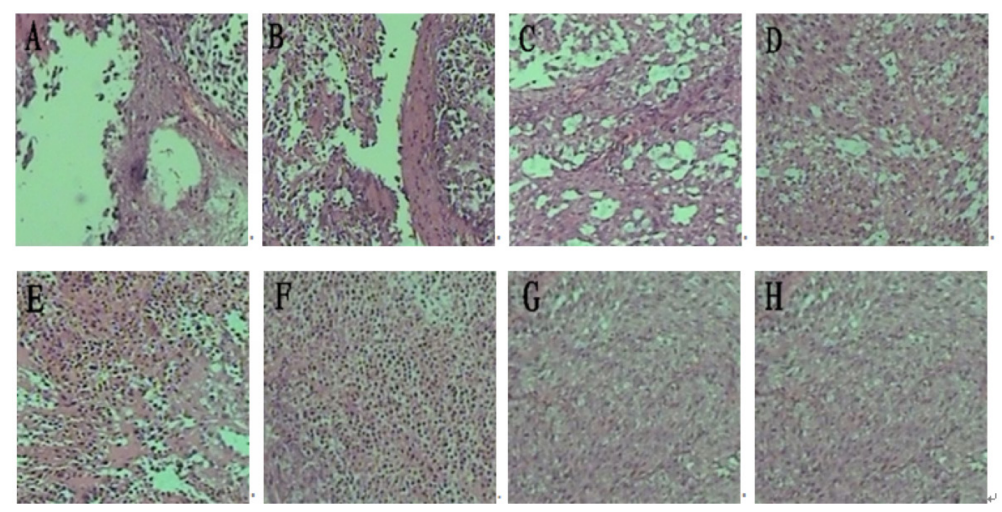

Figure 5: H\&E histological staining of tumor sections from mice injected with different formulations. A. DTIC-NPsDR5 mAb group. B. DTIC + DR 5 mAb group. C. DTIC-NPs group. D. DTIC group. E. DR5 mAb group. F. Blank-NPs-DR5 mAb group. G. Blank-NPs group. H. PBS group. 

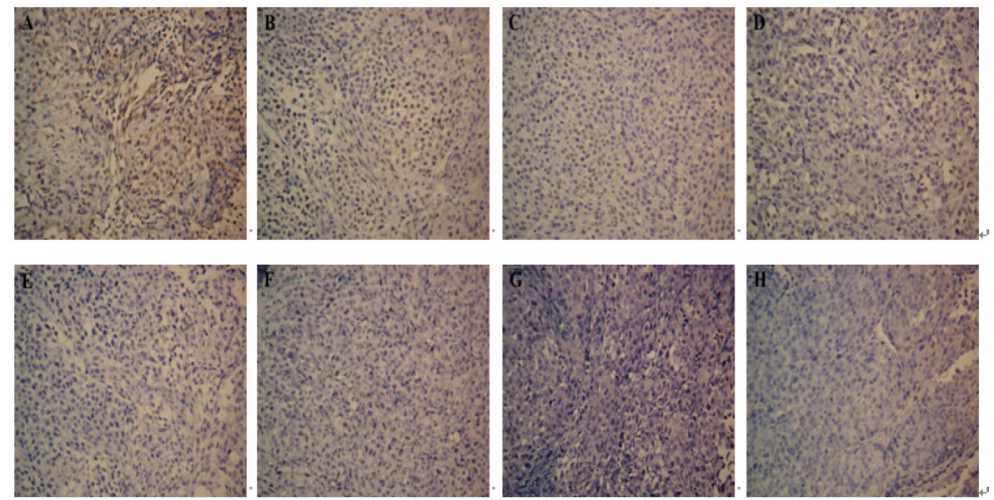

Figure 6: Apoptosis measurement of tumor sections from mice injected with different formulations: TUNEL assay $(\times \mathbf{2 0 0})$. A. DTIC-NPs-DR5 mAb group. B. DTIC + DR5 mAb group. C. DTIC-NPs group. D. DTIC group. E. DR5 mAb group. F. Blank-NPs-DR5 mAb group. G. Blank-NPs group. H. PBS group.

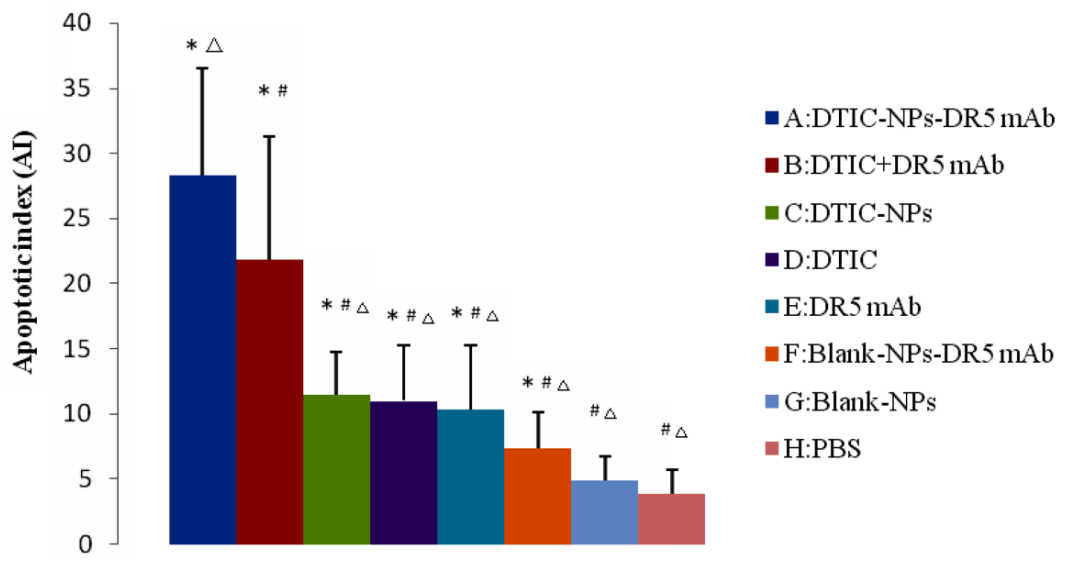

Figure 7: Apoptosis measurement of tumor sections from mice injected with different formulations: quantification of the apoptotic cells $(\mathbf{n}=\mathbf{6})$. The apoptotic index (AI) represents the number of TUNEL ${ }^{+}$cells per 100 cells $(\mathrm{n}=6)$. ${ }^{\#} p<0.05$, compared with DTIC-NPs-DR5 mAb (group A). ${ }^{\Delta} p<0.05$, compared with DTIC+DR5 mAb (group B). * $p<0.05$, compared with blank control group (group H).
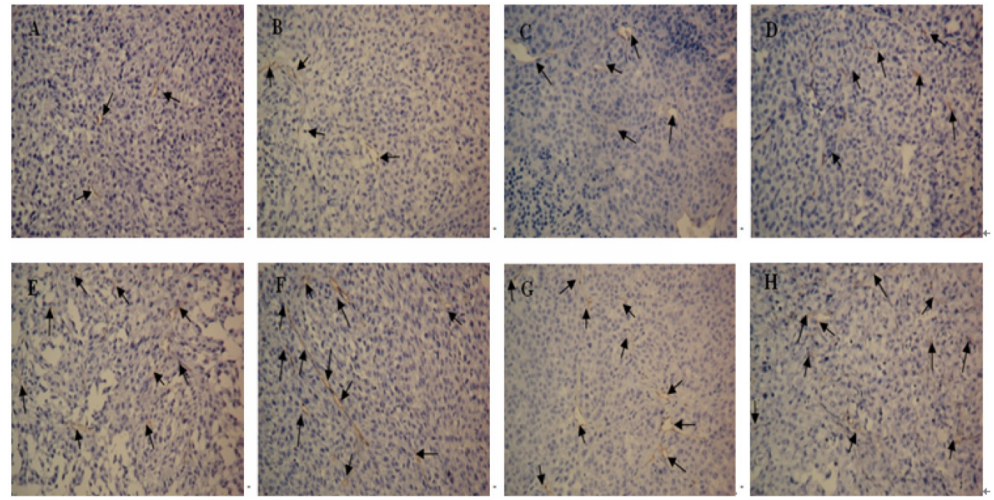

Figure 8: CD34 immunohistochemistry of tumor sections from mice after injection with different formulations: CD34 immunohistochemistry $(\times \mathbf{2 0 0})$. A. DTIC-NPs-DR5 mAb group. B. DTIC + DR5 mAb group. C. DTIC-NPs group. D. DTIC group. E. DR5 mAb group. F. blank-NPs-DR5 mAb group. G. blank-NPs group. H. PBS group. The arrow indicates the microvessel. 


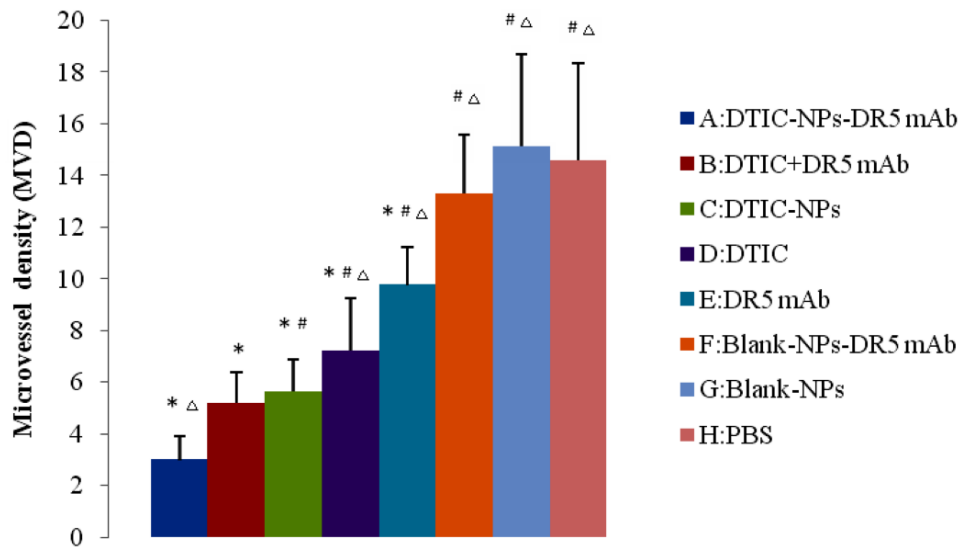

Figure 9: CD34 immunohistochemistry of tumor sections of mice after injection with different formulations: microvessel density (MVD) in the tumor sections (n=6). ${ }^{*} p<0.05$, compared with DTIC-NPs-DR5 mAb (group A). ${ }^{\Delta} p<0.05$, compared with DTIC+DR5 mAb (group B). * $p<0.05$, compared with blank control group (group H).

Table 4: Test results of the white blood cells number (WBC), alanine aminotransferase (ALT) data and creatinine clearance $(C R)$ of tumor-bearing nude mice after the treatment $(n=6)$

\begin{tabular}{|c|c|c|c|}
\hline Group & $\mathrm{WBC}\left(\times 10^{9} / \mathrm{L}\right)$ & $\operatorname{ALT}(u / L)$ & $\mathrm{CR}(\mu \mathrm{mol} / \mathrm{L})$ \\
\hline A & $2.77 \pm 0.29$ & $38.25 \pm 4.03$ & $9.33 \pm 2.34$ \\
\hline B & $2.31 \pm 0.46^{\text {*\# }}$ & $43.50 \pm 11.40^{* \#}$ & $7.00 \pm 2.19$ \\
\hline $\mathrm{C}$ & $2.57 \pm 0.36$ & $38.00 \pm 8.94$ & $8.18 \pm 3.66$ \\
\hline $\mathrm{D}$ & $2.50 \pm 0.33^{* \#}$ & $41.17 \pm 11.30^{* \#}$ & $9.33 \pm 1.51$ \\
\hline $\mathrm{E}$ & $2.92 \pm 0.54$ & $37.50 \pm 3.70$ & $8.17 \pm 2.48$ \\
\hline $\mathrm{F}$ & $3.18 \pm 0.84$ & $35.33 \pm 12.9$ & $7.25 \pm 0.50$ \\
\hline G & $3.40 \pm 0.98$ & $35.33 \pm 7.12$ & $10.00 \pm 1.83$ \\
\hline $\mathrm{H}$ & $3.51 \pm 1.54$ & $36.50 \pm 6.45$ & $9.50 \pm 1.82$ \\
\hline Normal control & $3.66 \pm 0.51$ & $35.66 \pm 5.27$ & $9.17 \pm 2.86$ \\
\hline
\end{tabular}

${ }^{*} p<0.05$, compared with blank control group (group H). ${ }^{*} p<0.05$, compared with normal control group (healthy nude mice)

surface can trigger apoptotic cell death providing additional tumoricidal effects on top of DTIC. Furthermore, DR5 $\mathrm{mAb}$ also acts as an active targeting device for the nanoparticles recognized by melanoma cells.

The distribution and accumulation results show that the unmodified NPs non-specifically distributed in various tissues, while the DR5 mAb-NPs more specifically accumulated in tumor tissue. The unmodified NPs possess a passive targeting property due to the EPR effect, which may lead to their accumulation in tumor tissue to a certain extent. The incorporation of DR5 mAb further improved the tumor targeting effect by recognizing and binding to the over-expressed TRAIL-R2 in melanoma cells. Therefore, the mAb-NPs can actively target the melanoma cells resulting in an increased level of intracellular delivery of the NPs.
The in vivo antitumor results indicated that the combination of DTIC and DR $5 \mathrm{mAb}$ has an enhanced tumoricidal effect, as the DTIC $+\mathrm{mAb}$ group, significantly inhibited tumor growth relative to the DTIC or DR5 mAb monotherapy or DTIC loaded in NPs. In a previous study, the combination of the DTIC + lexatumumab (agonistic TRAIL receptor -2 antibody) increased the sensitivity of tumor cells to the cytotoxic effect of DTIC or antibody, and reduced the growth of FEMX-1 xenografts in vivo compared with single drug [31]. The tumor volume of the mice treated with the DTIC-NPs-DR5 mAb formulation was significantly reduced compared to the DITC+ DR5 $\mathrm{mAb}(p<0.05)$, indicating that this pharmaceutical engineering not only obtained the combined antitumor effect of DITC+ DR5 mAb, but that active targeting of the delivery carrier provided an additional advantage. In 
addition, DTIC-NPs-DR5 mAb also showed a controlled release property, which further prolongs survival time.

$\mathrm{H} \& \mathrm{E}$ staining reveals that DTIC-NPs-DR5 mAb treatment significantly induced tumor cell necrosis and apoptosis, leading to superior suppression of tumor progression relative to other groups. TUNNEL assay results shows that the effect of Blank-NPs-DR5 $\mathrm{mAb}$ on tumor apoptosis was similar to that of DR5 mAb, indicating that the conjugation of DR5 mAb to the NPs has no effect on the activity of DR $5 \mathrm{mAb}$. In contrast to monotherapy with DTIC or DR5 mAb, the combination of DTIC and DR5 mAb (Groups A and B) shows an additive and synergistic effect on apoptosis, which may due to the induced apoptosis by the intrinsic pathway of DTIC and extrinsic pathway of DR5 mAb. The CD34 immunohistochemistry study was conducted to evaluate neovascularization. MVD in the DTIC-NPs-DR5 mAb group was relatively lower compared with other groups. This may be due to the cell necrosis and apoptosis caused by the combination of DTIC and DR5 mAb, thereby reducing tumor growth. The systemic toxicity results showed that DTIC caused the marrow and hepatic toxicity. When DTIC was encapsulated within the DTIC-NPsDR5 mAb formulation, its toxicity was reduced, possibly due to the reduced distribution of NPs in normal tissues by the active targeting effect and the decreased plasma concentration of DTIC through controlled drug release. DTIC showed antitumor effect, but had little effect on survival time, possibly due to its serious side-effects.

To summarize, we developed a novel dual function immuno-nanoparticle preparation, DTIC-NPs-DR5 $\mathrm{mAb}$, which contains DTIC on the inside and covalently conjugated with the DR5 mAb on the outside. In vivo and ex vivo studies indicates that DTIC-NPs-DR5 mAb have an improved therapeutic efficacy with lower toxicity. Therefore, these immuno-nanoparticles offer a promising formulation for the treatment of melanoma.

\section{MATERIALS AND METHODS}

\section{Materials}

DTIC was purchased from Suzhou Lixin Pharmaceutical Co. (Suzhou, China). Methoxy poly (ethylene glycol)-poly (lactide) (MPEG-PLA) (3000:30000, Mw=33,000) was obtained from Shandong Institute of Medical Instruments (Shangdong, China). Humanized anti-DR5 mAb and FITC-labeled anti-DR5 $\mathrm{mAb}$ were purchased from Santa Cruz Biotechnology, Inc. (CA, USA). The human malignant melanoma cell line A375 was maintained in the International Joint Cancer Institute, Second Military Medical University (Shanghai, China). Dulbecco's Modified Eagle's Medium and fetal bovine serum were purchased from Hyclone (Logan, UT, USA). CD34 mouse anti-human monoclonal antibody was obtained from Maixin Biotechnology Development Co., Ltd. (Fuzhou, China). TUNEL apoptosis assay kit and IHC detection kit were purchased from Boehringer Mannheim GmbH. (Mannheim, Germany). Male BALB/c mice (4-6 weeks old) weighing 19-20 g were obtained from Shanghai Experimental Animal Center of the Chinese Academy of Sciences (Shanghai, China), and housed in groups under standard housing conditions. All experimental procedures were approved by the Animal Care and Use Committee of the Second Military Medical University. All other chemicals used in this work were of analytical grade and used as received.

\section{Preparation of nanoparticles}

DTIC-NPs-DR5 mAb were prepared by a two-step procedure as described previously [30]. Briefly, DTICNPs were prepared using the double emulsion (w/o/w) and solvent evaporation method. Subsequently, DR5 mAb was conjugated to DTIC-NPs with EDC as the coupling agent. After preparation, the product was lyophilized. Control preparations, including DTIC-NPs, Blank-NPs-DR5 mAb, PE-NPs (phycoerythrin-loaded NPs), and PE-NPs-DR5 mAb-FITC (DTIC-NPs conjugated with FITC-labeled DR5 mAb) were obtained in a similar manner.

\section{Establishment of A375 BALB/c nude mouse tumor model}

Tumor implantation was carried out by injecting $1 \times 10^{6}$ A 375 cells $\left(5.0 \times 10^{6}\right.$ cells $/ \mathrm{ml}, 0.2 \mathrm{ml}$ per mouse $)$ into the back subcutaneous region of each BALB/c nude mouse. The procedure was performed in a laminar flow hood using aseptic techniques. Tumor growth after implantation was monitored daily until Day 14 . Mice with tumor size of approximately $150 \mathrm{~mm}^{3}$ were selected for the study.

\section{Evaluation of in vivo tumor targeting effect}

To evaluate the in vivo real-time distribution and tumor targeting effect of DTIC-NPs-DR5 mAb in tumorbearing nude mouse, 5 A375 malignant melanoma bearing nude mice were randomly selected and anesthetized. One mouse was injected intravenously with PE-NPs-DR5 $\mathrm{mAb}-$ FITC $(5 \mathrm{mg} / \mathrm{ml}, 0.2 \mathrm{ml})$ via the tail vein, and another untreated mouse was used as the negative control. Both mice were placed in the observation room of the IVIS Lumina II small animal in vivo imaging system after 1, 3, 6,10 and $24 \mathrm{~h}$ for real-time observation. The other three mice were given intravenous Blank-NPs, PE-NPs and PENPs-DR5 mAbs-FITC $(5 \mathrm{mg} / \mathrm{ml}, 0.2 \mathrm{ml})$ via the tail vein. After $10 \mathrm{~h}$, all mice were sacrificed and major organs and the tumor were removed immediately for imaging using the same imaging system. 


\section{Animal groups and dosage regimens}

To examine the therapeutic effect of DTIC-NPsDR5 mAb in vivo, 48 A375 malignant melanoma-bearing nude mice were randomly divided into eight groups of 6 mice as follows: Group A (DTIC-NPs-DR5 mAb treatment group); Group B (DTIC + DR5 mAb treatment group); Group C(DTIC-NPs treatment group); Group D (DTIC treatment group); Group E (DR5 mAb treatment group); Group F (Blank-NPs-DR5 mAb treatment group); Group G (Blank-NPs treatment group); Group H (Blank control group). DTIC-NPs-DR5 mAb were administered once at a dosage of $2.5 \mathrm{mg} / \mathrm{kg}$ by tail vein injection on Days 1,3 , 5 and 7 . The dosage of the other treatment groups was adjusted to be equal to DTIC or DR5 mAb in the DTICNPs-DR5 mAb group. Group H received PBS as a blank control.

\section{Determination of in vivo antitumor effect}

After dose administration, the therapeutic effect was evaluated by tumor growth suppressive and survival time. The tumor size was measured with a caliper every other day. Tumor volumes were calculated by the formula: $\mathrm{V}=$ length $\times$ width $^{2} / 2$. The mice were sacrificed by cervical dislocation and blood was collected after 16 day-treatment. Tumors were stripped and weighed to calculate the inhibition rate using the following formula: tumor weight inhibition rate $(\%)=$ (average tumor weight of the PBS control group - average tumor weight of drug treatment group / average tumor weight of the PBS control group) $\times 100 \%$.

Another 80 tumor-bearing mice were randomly divided into 8 groups and then treated as above. The mice were observed daily until death. The survival time was recorded and analyzed.

\section{Histochemical and immunohistochemical analysis of tumor tissue}

The tumor tissues, obtained from the in vivo experiment, were fixed, dehydrated, embedded, and cut into serial sections, one of the sections was stained with haematoxylin and eosin (H\&E) using a standard protocol for tissue histological examination under light microscope.

Tumor apoptosis was measured by the TUNEL assay. Briefly, paraffin sections of the tumor tissue from each group were dewaxed with xylene and washed with PBS ( $\mathrm{pH}$ 7.4) three times. The sections were digested with proteinase $\mathrm{K}$ for $15 \mathrm{~min}$, blocked by $3 \% \mathrm{H}_{2} \mathrm{O}_{2}$ for $30 \mathrm{~min}$, added with the TUNEL mixed reaction liquid and then incubated a wet box at $40{ }^{\circ} \mathrm{C}$ overnight. After a 15 min incubation with POD anti-fluorescein antibody at $37^{\circ} \mathrm{C}$, the sections were visualized using Converter-POD with diaminobenzidine (DAB) and subsequently counterstained with hematoxylin. The sections were observed under a light microscope and the TUNEL ${ }^{+}$cells, which distributed homogeneously within sight were counted. The apoptotic index (AI) represents the number of TUNEL ${ }^{+}$ cells per 100 cells.

To investigate the intratumoral microvessel density (MVD), three tumor tissue sections were used for immunohistochemical staining (S-P method) with a monoclonal anti-mouse CD34 antibody. The staining was carried out using routine operating procedures. The microvessels were confirmed as CD34 positive staining area and counted under microscope. In case of the unknown pathological features, the highest measured vascular density area was selected at low magnification. Microvessels were counted from five fields of view, and then the average value of microvessel counts was calculated.

\section{Toxicity study}

To assess the adverse effect of different formulations, the alanine aminotransferase (ALT), creatinine (Cr), and white blood cell count (WBC) in the blood of the treated mice were measured in the hospital clinical laboratory using routine techniques. Healthy nude mice were used as normal control.

\section{Data analysis}

Data are presented as mean \pm SD. Statistical significance was determined using two-sample $t$ test and the analysis of variance (ANOVA) using the SPSS19.0 software with $\mathrm{P}<0.05$ as a significance level. Survival analyze was conducted using the Kaplan-Meier method and the comparison of groups was analyzed with the Logrank.

\section{ACKOWLEDGMENTS}

We acknowledge the financial support of the National Natural Science Foundation (No.81201809, $81472349,81400654,81302122,8140219081503338$ and 81400489), Zhejiang Provincial Natural Science Foundation (No.LQ12H30005) and Public Welfare Technology Application Research Project (No.2015C37125), Shanghai Natural Science Foundation (No.14ZR1433300 and 15ZR1412700), Zhejiang Provincial Education Department (No.Y201330031 and Y201431468), Science and Technology Projects of Jiaxing (No.2012AY1075-3 and 2015AY23064), Key Scientific and Technological Innovation Team of Jiaxing (2012) (Technological Innovation Team of Research and Development about Natural Medicine and Health Food), Project of Science and Technology Innovation for Undergraduate of Zhejiang Province (No.2015R417001), Key project of Jiaxing University students research training program (No.851714068 and 851715062), and the Zhejiang Provincial $12^{\text {th }}$ Five-year Plan for University Key Academic Subject of China (Pharmacology). 


\section{CONFLICTS OF INTEREST}

The authors declare no competing financial interest.

\section{REFERENCES}

1. Rigon RB, Oyafuso MH, Fujimura AT, Gonçalez ML, Prado AH, Gremião MP D, Chorilli M. NanotechnologyBased Drug Delivery Systems for Melanoma Antitumoral Therapy: A Review. BioMed research international. 2015; doi: $10.1155 / 2015 / 841817$.

2. Niezgoda A, Niezgoda P, Czajkowski R Novel Approaches to Treatment of Advanced Melanoma: A Review on Targeted Therapy and Immunotherapy. BioMed research international. 2015; doi: 10.1155/2015/851387.

3. Garbe C, Eigentler TK. Diagnosis and treatment of cutaneous melanoma: state of the art 2006. Melanoma research. 2007; 17:117-127.

4. Murphy MJ. Diagnostic and prognostic biomarkers and therapeutic targets in melanoma. Totowa, NJ, Humana, 2012.

5. Eigentler TK, Caroli UM, Radny P, Garbe C. Palliative therapy of disseminated malignant melanoma: a systematic review of 41 randomised clinical trials. Lancet oncology. 2003; 4:748-759.

6. Quirin C, Mainka A, Hesse A, Nettelbeck DM. Combining adenoviral oncolysis with temozolomide improves cell killing of melanoma cells. International journal of cancer. 2007; 121:2801-2807.

7. Lillehammer T, Engesaeter BO, Prasmickaite L, Maelandsmo GM, Fodstad O, Engebraaten O. Combined treatment with Ad-hTRAIL and DTIC or SAHA is associated with increased mitochondrial-mediated apoptosis in human melanoma cell lines. Journal of gene medicine. 2007; 9:440-451.

8. Xie T, Nguyen T, Hupe M, Wei ML. Multidrug resistance decreases with mutations of melanosomal regulatory genes. Cancer research. 2009; 69:992-999.

9. La Porta CA. Drug resistance in melanoma: new perspectives. Current medicinal chemistry. 2007; 14:387-391.

10. McNulty SE, del Rosario R, Cen D, Meyskens FLJr, Yang S. Comparative expression of NFkappaB proteins in melanocytes of normal skin vs. benign intradermal naevus and human metastatic melanoma biopsies. Pigment cell research. 2004; 17:173-180.

11. Merino D, Lalaoui N, Morizot A, Solary E, Micheau O. TRAIL in cancer therapy: present and future challenges. Expert opinion on therapeutic targets. 2007; 11:1299-1314.

12. Trivedi R, Mishra DP. Trailing TRAIL Resistance: Novel Targets for TRAIL Sensitization in Cancer Cells. Frontiers oncology. 2015; 5:69.

13. Daniel PT, Wieder T, Sturm I, Schulze-Osthoff K. The kiss of death: promises and failures of death receptors and ligands in cancer therapy. Leukemia. 2001; 15:1022-1032.
14. Suzuki-Karasaki Y, Suzuki-Karasaki M, Uchida M, Ochiai T. Depolarization Controls TRAIL-Sensitization and Tumor-Selective Killing of Cancer Cells: Crosstalk with ROS. Frontiers oncology. 2014; 4:128.

15. Falschlehner C, Ganten TM, Koschny R, Schaefer U, Walczak H. TRAIL and other TRAIL receptor agonists as novel cancer therapeutics. Advances in experimental medicine and biology. 2009; 647:195-206.

16. Li J, Shen Q, Peng R, Chen R, Jiang P, Li Y, Zhang L, Lu J. Plumbagin enhances TRAIL-mediated apoptosis through up-regulation of death receptor in human melanoma A375 cells. Journal of huazhong university of science and technology-medical sciences. 2010; 30:458-463.

17. Mao ZG, Jiang CC, Yang F, Thorne RF, Hersey P, Zhang XD. TRAIL-induced apoptosis of human melanoma cells involves activation of caspase-4. Apoptosis. 2010; 15: 1211-1222.

18. Chen Q, Ray S, Hussein MA, Srkalovi G, Almasan A. Role of Apo2L/TRAIL and Bcl-2-family proteins in apoptosis of multiple myeloma. Leukemia \& lymphoma. 2003; 44: 1209-1214.

19. Zhang XD, Franco AV, Nguyen T, Gray CP, Hersey P. Differential localization and regulation of death and decoy receptors for TNF-related apoptosis-inducing ligand (TRAIL) in human melanoma cells. Journal of immunology. 2000; 164:3961-3970.

20. Eberle J, Fecker LF, Hossini AM, Kurbanov BM, Fechner H. Apoptosis pathways and oncolytic adenoviral vectors: promising targets and tools to overcome therapy resistance of malignant melanoma. Experimental dermatology. 2008; 17:1-11.

21. Ichikawa K, Liu W, Zhao L, Wang Z, Liu D, Ohtsuka T, Zhang H, Mountz JD, Koopman WJ, Kimberly RP, Zhou T. Tumoricidal activity of a novel anti-human DR5 monoclonal antibody without hepatocyte cytotoxicity. Nature medicine. 2001; 7:954-960.

22. Cretney E, Takeda K, Smyth MJ. Cancer: novel therapeutic strategies that exploit the TNF-related apoptosis-inducing ligand (TRAIL)/TRAIL receptor pathway. International journal of biochemistry and cell biology. 2007; 39:280-286.

23. Georgakis GV, Li Y, Humphreys R, Andreeff M, O'Brien S, Younes M, Carbone A, Albert V, Younes A. Activity of selective fully human agonistic antibodies to the TRAIL death receptors TRAIL-R1 and TRAIL-R2 in primary and cultured lymphoma cells: induction of apoptosis and enhancement of doxorubicin- and bortezomibinduced cell death. British journal of haematology. 2005; 130:501-510.

24. Motoki K, Mori E, Matsumoto A, Thomas M, Tomura T, Humphreys R, Albert V, Muto M, Yoshida H, Aoki M, Tamada T, Kuroki R, Yoshida H, Ishida I, Ware CF, Kataoka $\mathrm{S}$. Enhanced apoptosis and tumor regression induced by a direct agonist antibody to tumor necrosis factor-related apoptosis-inducing ligand receptor 2. Clinical cancer research. 2005; 11:3126-3135. 
25. Pukac L, Kanakaraj P, Humphreys R, Alderson R, Bloom M, Sung C, Riccobene T, Johnson R, Fiscella M, Mahoney A, Carrell J, Boyd E, Yao XT, Zhang L, Zhong L, von Kerczek A, Shepard L, Vaughan T, Edwards B, Dobson C, Salcedo T, Albert V. HGS-ETR1, a fully human TRAILreceptor 1 monoclonal antibody, induces cell death in multiple tumour types in vitro and in vivo. British journal of cancer. 2005; 92:1430-1441.

26. Duiker EW, Mom CH, de Jong S, Willemse PH, Gietema JA, van der Zee AG, de Vries EG. The clinical trail of TRAIL. European journal of cancer. 2006; 42:2233-2240.

27. Attard G, Kitzen J, Blagden S, Fong PC, Pronk LC, Zhi J, Zugmaier G, Verweij J, de Bono JS, de Jonge M. A phase Ib study of pertuzumab, a recombinant humanised antibody to HER2, and docetaxel in patients with advanced solid tumours. British journal of cancer. 2007; 97:1338-1343.

28. Gallamini A, Zaja F, Patti C, Billio A, Specchia MR, Tucci A, Levis A, Manna A, Secondo V, Rigacci L, Pinto A, Iannitto E, Zoli V, Torchio P, Pileri S, Tarella C. Alemtuzumab (Campath-1H) and CHOP chemotherapy as first-line treatment of peripheral T-cell lymphoma: results of a GITIL (Gruppo Italiano Terapie Innovative nei Linfomi) prospective multicenter trial. Blood. 2007; 110:2316-2323.

29. Kowalczyk A, Gil M, Horwacik I, Odrowaz Z, Kozbor D, Rokita H. The GD2-specific 14G2a monoclonal antibody induces apoptosis and enhances cytotoxicity of chemotherapeutic drugs in IMR-32 human neuroblastoma cells. Cancer letter. 2009; 281:171-182.

30. Ding B, Wu X, Fan W, Wu Z, Gao J, Zhang W, Ma L, Xiang W, Zhu Q, Liu J, Ding X, Gao S. Anti-DR5 monoclonal antibody-mediated DTIC-loaded nanoparticles combining chemotherapy and immunotherapy for malignant melanoma: target formulation development and in vitro anticancer activity. International journal of nanomedicine. 2011; 6:1991-2005.

31. Engesaeter B, Engebraaten O, Florenes VA, Maelandsmo GM. Dacarbazine and the agonistic TRAIL receptor-2 antibody lexatumumab induce synergistic anticancer effects in melanoma. PLOS ONE. 2012; 7: 45492. 